\title{
Joining a Mathematical Research Community
}

\section{Leslie Hogben and T. Christine Stevens}

Mathematics has always had research communities, although that terminology was not used and they were usually not as open as they are today. A research community can serve as a professional neighborhood, providing friendship (networking) and access to local knowledge (professional development). Research communities play an important role in keeping people engaged in research, helping them succeed in their careers, and shaping the discipline of mathematics. Traditional research communities have frequently been unintentionally exclusive, often being based on informal power networks and sometimes requiring substantial resources for travel.

In many cases those underrepresented in research mathematics, such as women, minorities, persons with disabilities, and faculty in departments without doctoral programs, have been even more underrepresented in research communities. To ensure that all mathematicians have access to the benefits of research communities, there has been an intentional effort over the past ten to twenty years to build strong research communities with well-publicized open application processes. All four programs profiled here welcome applications and are clear about who is eligible. Each is supported by grants from the National Science Foundation and other organizations, and such

Leslie Hogben is Dio Lewis Holl Chair in Applied Mathematics, professor of mathematics, and associate dean for graduate studies and faculty development at the College of Liberal Arts and Sciences at Iowa State University, and associate director for diversity at the American Institute of Mathematics. Her email address is hogben@aimath.org.

T. Christine Stevens is associate executive director for meetings and professional services at the AMS. Her email address is tcs@ams.org.

For permission to reprint this article, please contact: reprint-permission aams.org.

DOI: https://dx.doi.org/10.1090/noti 1913 support has played an important role in the development of research communities with open application processes.

By a research community, we mean a group of mathematicians with some common interests who collaborate on research with some other members of the community and who support each other professionally, but which is larger than a group of co-authors even if they work together on an ongoing basis. This definition is intentionally rather loose and broad, and is perhaps best illustrated by the four quite different research community programs we have chosen to profile. Three of them-the Mathematics Research Communities (MRC), the Research Collaboration Conferences for Women (RCCW), and the Park City Math Institute (PCMI) - build a research community around a single research theme. The fourth, the Research Experiences for Undergraduate Faculty (REUF), builds a community of researchers who support research with undergraduate students, with subgroups focused on different research topics. MRC, RCCW, and REUF have limited target audiences, serving, respectively, junior researchers, women, and faculty at undergraduate institutions. PCMI is vertically integrated, bringing together groups of researchers, graduate students, undergraduate faculty, undergraduate students, and secondary school teachers. MRC, RCCW, and REUF are each one week long and include continuation activities, whereas PCMI is three weeks long. PCMI is the oldest, beginning in 1991 as a Regional Geometry Institute and as PCMI in 1995, with the others operating first in 2008 (in at least some preliminary form). Each has a major research emphasis (and produces published research), but also includes professional development and networking opportunities. Each includes multiple groups of collaborators. Each MRC has either twenty or forty participants, and an RCCW usually has thirty to fifty people, with several workshops each year. REUF has about twenty participants per annual workshop, 
and PCMI has about 300 people in several subprograms that meet in parallel at the same site each year. PCMI, REUF, and MRC all meet in the summer, as do many but not all RCCW workshops.

Here we have profiled four research community programs, but there are many others. For example, the NSF Mathematical Sciences Institutes offer many research opportunities for which there are open applications [8], and some of these create research communities. Additional information about opportunities to join a mathematical research community (and about other professional development opportunities and communities, including those that support teaching) can be found in the Mathematics Opportunities section of each month's Notices [9] and on the listing of Awards, Fellowships, and Other Opportunities on the AMS website [2].

\section{Mathematics Research Communities (MRC)}

The AMS developed the Mathematics Research Communities (MRC) as a national program to support and guide young mathematicians as they begin their research careers. Currently serving 120 early career mathematicians each year, the program fosters the creation of collaborative research groups and provides the participants with mentoring from leading scholars in their fields. Each year, three to five mathematical research areas are chosen as topics for MRCs, each of which includes twenty or forty participants.

An MRC is a year-long experience that begins with an intensive one-week conference in the summer. A typical MRC conference includes little or no lecturing, with the participants instead spending most of their time working in groups on open problems related to the topic of the conference. To make this format possible, the participants may interact electronically with the organizers before the conference and do some background preparation. The

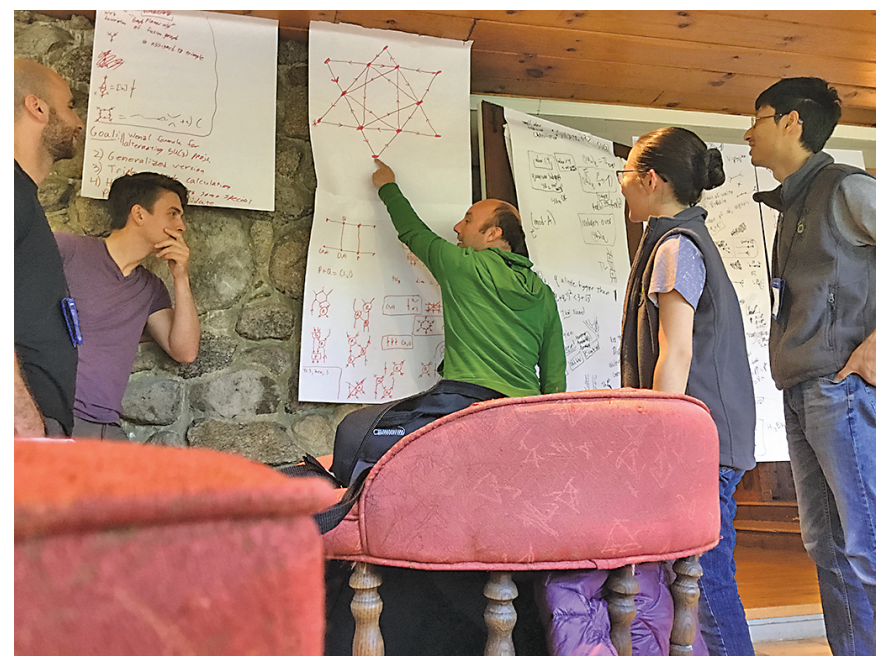

MRC participants spend most of their time working collaboratively on research problems, as this group did in 2018. week also includes professional development sessions that address the concerns of graduate students and recent PhDs, such as applying for jobs, choosing appropriate venues for publications, and writing grant proposals. The conferences are currently held at Whispering Pines Conference Center in West Greenwich, Rhode Island.

The MRC experience continues at the Joint Mathematics Meetings (JMM) the following January and in research collaborations throughout the year. Three of the participants in each summer conference are invited to organize an AMS Special Session at the JMM on the topic of the MRC. Although some of the talks in this Special Session are given by the MRC participants themselves, this event also serves as an opportunity to expand the research community to include people at all career stages who did not attend the summer conference. Both before and after the JMM, participants are eligible for travel funding for follow-up collaborations that enable them to finish the projects that they launched at the summer conference and to initiate new ones. After their year of formal MRC participation is over, MRC alumni can request funding for "microconferences," which are gatherings of ten to fifteen mathematicians that advance the mathematical work emanating from their original MRC summer conference.

Through 2018, there have been forty different MRCs on topics that range throughout the mathematical sciences [3]. Pending funding, there will be five MRCs in 2020, on dynamical models of the ecology of infectious diseases, using combinatorics and linear algebra to attack inverse problems, combinatorial applications of computational topology and algebraic geometry, analysis in metric spaces, and new problems in several complex variables. The application portal for 2020 will open in the summer of 2019.

MRC funding is open to early career individuals who are affiliated with US institutions or are US citizens or permanent residents. A few international participants may be accepted. Applicants from academic institutions of all types are welcome, as well as from private industry and government laboratories and agencies. The goal is to create a collaborative research community that is vibrant, productive, and diverse. All participants are expected to be active in the full array of MRC activities-the summer conference, Special Sessions at the Joint Mathematics Meetings, and follow-up collaborations.

Since its inception in 2008, the MRC program has helped some 1,300 young mathematicians to launch their research careers. Their collaborations are productive, with many reporting publications that emerged from their work at the MRCs. Here are some comments that members of the 2013 cohort made in 2018 when they were asked to reflect upon the impact of their MRC experience:

- My MRC mentor was my mentor for my first postdoc, we wrote a paper that was published in Geometry and Topology, and we continue to talk to this day, even after 
I have moved to another institution.... MRC had a positive impact on the social aspect of my career too, as I continue to see many of my fellow participants at conferences and meetings.

- The MRC has played an important role in my career. The one week of intense mathematical activity shaped my research plans for the coming years. The program led to two papers and equally importantly, it gave me the opportunity to interact with several active postdoctoral researchers and graduate students working in tropical geometry. The mentoring provided by the two experts was very valuable.

The organizers also praise the format of the MRCs, often commenting on the satisfaction they derive from "seeing the participants from such different backgrounds gel and start making real serious progress on the problems." As a 2018 organizer put it:

I am really glad that we went "all in" with the philosophy of the MRC program and devoted as much of the scheduled time to group work as possible. This was a very different approach than most of us were used to, but we got a lot of strong feedback during the week about how participants preferred the approach to other summer schools, etc. they had done.

More information about organizing an MRC can be found in [10]. Pending funding, proposals for the 2021 MRC conferences will be due in August 2019.

Who: Graduate students, postdocs, and early career faculty-people with $\mathrm{PhD}$ age in the interval $[-2,5]$. In each given week, there is either one MRC with forty participants or two with twenty each, for a total of 120 participants per year.

What: One-week summer conference, Special Session at the Joint Mathematics Meetings (JMM), and follow-up collaborations.

When: Summer conference is in June; JMM is in January.

Where: For 2018 and 2019, the conference site was the Whispering Pines Conference Center in West Greenwich, RI.

Website: www.ams.org/programs/researchcommunities/mrc

Apply: Online via MathPrograms.org. The application portal for each summer conference opens in the previous summer; applications are due in February.

\section{Park City Mathematics Institute (PCMI)}

The Park City Mathematics Institute (PCMI) builds a vertically integrated community by annually running several parallel programs around a chosen research theme. Its constituent programs include the Research Program (RP), Graduate Summer School (GSS), Undergraduate Faculty Program (UFP), Undergraduate Summer School (USS), and Teacher Leadership Program (TLP), each lasting for three weeks, and the Workshop on Equity and Mathematics Education (WEME), which takes place during the first of the three weeks. Participants engage in program-specific activities but also interact through social and cross-program academic activities. Promoting such interaction between participants from across the entire spectrum of the mathematics profession is one of the key goals of PCMI, which is an outreach effort of the Institute for Advanced Study (IAS) and is now in its twenty-ninth year.

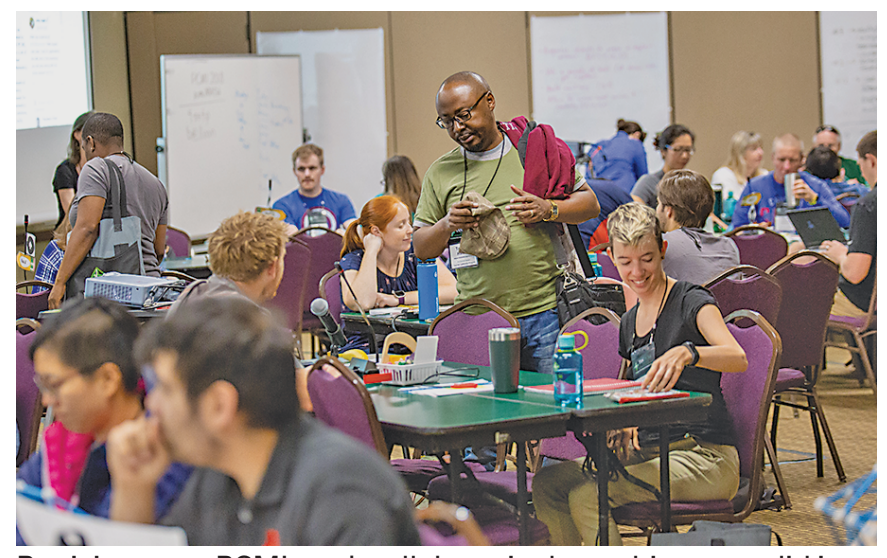

Participants at PCMI work collaboratively, as this group did in 2018.

The research theme for PCMI 2020 is "Number Theory Informed by Computation," organized by Jennifer Balakrishnan (Boston University), Kristin Lauter (Microsoft Research), Bjorn Poonen (MIT), and Akshay Venkatesh (IAS and Stanford). Research themes from the recent past include "Quantum Field Theory and Manifold Invariants" (2019), "Harmonic Analysis" (2018), "Random Matrices" (2017), and "The Mathematics of Data" (2016).

The GSS consists of several mini-courses delivered by lecturers selected by the organizers of that year's research theme in consultation with the PCMI Steering Committee. The courses range from relatively introductory to advanced, and TA sessions accompanying each lecture help students keep up with the rapid development of material. The RP is a research workshop with seminar talks and plenty of time for participants to work together or form new collaborations. The organizers invite a core of senior researchers to the RP, but applications to this program are also welcomed from researchers at all levels post-PhD. Many RP participants attend the graduate mini-courses, and conversely, 
many graduate students attend RP seminars and interact closely with the researchers in attendance. One job of the Steering Committee and organizers is to make sure that RP attendees do not regard this as "just another conference" where they talk to only a handful of people, but instead talk with participants from across PCMI.

The UFP provides an opportunity for faculty at undergraduate institutions to spark their research, as well as for any university faculty member to engage with themes related to undergraduate education. For example, the UFP participants in 2016 spent the session under the direction of Richard De Veaux (Williams College) designing an undergraduate data science curriculum, which was later published [7]. By contrast, the 2019 UFP session provided an intensive course on the topology of 4-manifolds led by John Etnyre (Georgia Tech) and Paul Melvin (Bryn Mawr), to enable participants to do computational research in this area (with or without undergraduate students). The core of the USS is a set of two parallel lecture courses for undergraduates related to that year's research theme. This is accompanied by opportunities for these students to engage in small-group open-ended research projects, with one another or with other PCMI participants. USS participants are also able to interact with graduate students and faculty from many universities, which leads to more informed decisions about graduate programs to which they might apply.

The TLP is a high-level professional development opportunity for $\mathrm{K}-12$ teachers from around the country. The theme of the WEME changes each year, but focuses broadly on equity and social justice issues as they relate to mathematics education and the mathematics profession.

By building a vertically integrated research community, PCMI creates meaningful interactions between members from different parts of the profession, in particular the mentoring of students and younger researchers by more established researchers. Some hint of participant experience is conveyed by the following quotes:

- Extended period of time with many of the same people allows you to get beyond the "getting to know someone" stage and have "substantive conversations," not only about actual math but also in terms of making connections with people you would not otherwise have heard about. This is a very good thing. (2018 RP)

- I got overwhelming support from professors, fellow undergraduates, and teachers I met. I got so much out of PCMI that it would be impossible to imagine it was only 3 weeks long. (2018 USS)
Who: RP: Researchers (60), GSS: Graduate students (80), USS: Undergraduate students (45), UFP: Faculty at undergraduate colleges and universities (15), WEME: Faculty (10), TLP: K-12 teachers (60).

What: Three-week summer session (WEME takes place only during the first week of PCMI).

When: Summer (the first three weeks of July).

Where: Park City, UT.

Website: https://www. ias . edu/pcmi

Apply: Online through the PCMI website. The application page is open from early November; applications for the RP, GSS, UFP, and TLP are due in mid-January; applications for the USS and WEME are typically due a few weeks later.

\section{Research Collaboration Conferences for Women (RCCW)}

The Association for Women in Mathematics (AWM) nurtures the building of Research Networks (RNs), which are research communities of women in particular mathematical areas. An RN usually begins with a Research Collaboration Conference for Women (RCCW), which is itself a research community, and an RN may later welcome more new members through additional RCCWs. Here we focus on the RCCW and its subsequent activities because an RN usually grows out of an RCCW, and participating in an RCCW is the way participants join an RN.

Each RCCW is a one-week workshop held at a mathematics institute where junior and senior women collaborate on specific research projects in one area. Women who are junior faculty, postdocs, and advanced graduate students can apply to participate in an RCCW through the host institute. Each participant is placed into a research group led by one or more senior women who plan the research projects in advance. The week of the RCCW is spent doing research collaboratively in teams. A few of the RCCWs have included some male participants, but the majority of

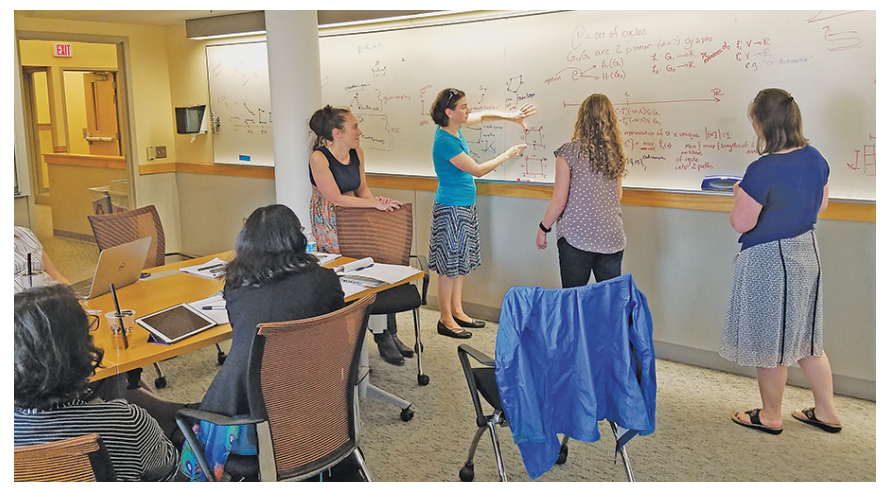

RCCW participants spend most of their time in working groups, such as this group at the Institute for Mathematics and its Applications in 2016. 
participants and the leaders are female. To date there are nineteen RNs [6], with five of them scheduled to have their first RCCWs during the next few months.

In most cases the research is only started at the RCCW, and the group continues working together after the conference via electronic communication and follow-up meetings, which include special sessions at the AWM workshops at the Joint Mathematics Meetings and the SIAM Annual Conference and at the AWM Research Symposium (held every two years). In addition to completing a research paper, this continued collaboration helps to form lasting bonds and build a community. Each RCCW is encouraged to publish an associated Proceedings volume of the AWMSpringer Series [4], and most do so.

RCCWs and RNs were designed to address the serious underrepresentation of women in mathematical research. The first RCCW was in number theory and was held at the Banff International Research Station (BIRS) in 2008 and led to the creation of the Women In Numbers (WIN) network [12], which has now run six RCCWs and published five volumes of research papers [13]. The success of the WIN network inspired the RCCW and RN model.

RCCWs have been hosted at institutes all over the world including BIRS, Institute for Computational and Experimental Research in Mathematics (ICERM), Institute for Pure and Applied Mathematics (IPAM), Institute for Mathematics and its Applications (IMA), American Institute of Mathematics (AIM), Mathematical Biosciences Institute (MBI), Centre International de Rencontres Mathématiques (France), Lorentz Center (Netherlands), and Nesin Mathematical Village (Turkey).

RCCW participants have been very enthusiastic about the experience, with one commenting:

The workshop was fantastic. The collaborative and supportive atmosphere was totally different than any other workshop I've attended, in the most wonderful way. I think we all felt comfortable, respected and valued as mathematicians.

Thus far we have described opportunities to participate in an RCCW that has already been organized. There are also opportunities to initiate a new RCCW and develop an $\mathrm{RN}$. The AWM ADVANCE Project provides mentorship and support to organizers wishing to create an RN by organizing its first RCCW, including help finding a conference venue, help developing and submitting a conference proposal, and help soliciting travel funding for participants [5].
Who: Women (faculty, postdocs, often advanced graduate students, usually thirty to fifty per workshop).

What: One-week workshop, subsequent special session at the AWM workshop at the Joint Mathematics Meetings or the SIAM Annual Conference and at the AWM Research Symposium.

When: Dates vary.

Where: A mathematical sciences institute.

Website: https: //awmadvance.org/rccws/

Apply: Online through the host institute (upcoming RCCWs are listed on the RCCW website under Year 5).

\section{Research Experiences for Undergraduate Faculty (REUF)}

The Research Experiences for Undergraduate Faculty (REUF) program builds a community of researchers who support undergraduate research by engaging faculty in departments without doctoral programs in small research groups as part of an annual one-week workshop held at the American Institute of Mathematics (AIM) or at the Institute for Computational and Experimental Research in Mathematics (ICERM). Since it began in 2008, more than 250 undergraduate researchers have been supervised by REUF alumni.

REUF models an undergraduate research experience by throwing participants into research problems for which they have limited background knowledge. They work in research teams that are led by senior faculty with expertise engaging undergraduates in research, and the topics are accessible to undergraduates. As undergraduate researchers often do, research groups frequently begin by experimenting and making conjectures, initially rediscovering known results, acquiring background along the way, and eventually discovering new results. Most of the time at the workshop is spent doing research in teams, but there are also wholegroup discussions related to undergraduate research. One participant commented:

I have greatly benefited from the variety of problems described, the discussion on the logistics of running an undergraduate research program, and the camaraderie with other liberal arts faculty. This has easily been the most worthwhile workshop or conference I've ever attended.

REUF was designed to enhance the ability of faculty at undergraduate colleges and universities to engage their students in research, but has become much more, involving faculty participants in long-term research collaborations, developing expertise in new research areas that have problems accessible to undergraduates, and helping them rediscover the joy of mathematics. Faculty come to REUF in 


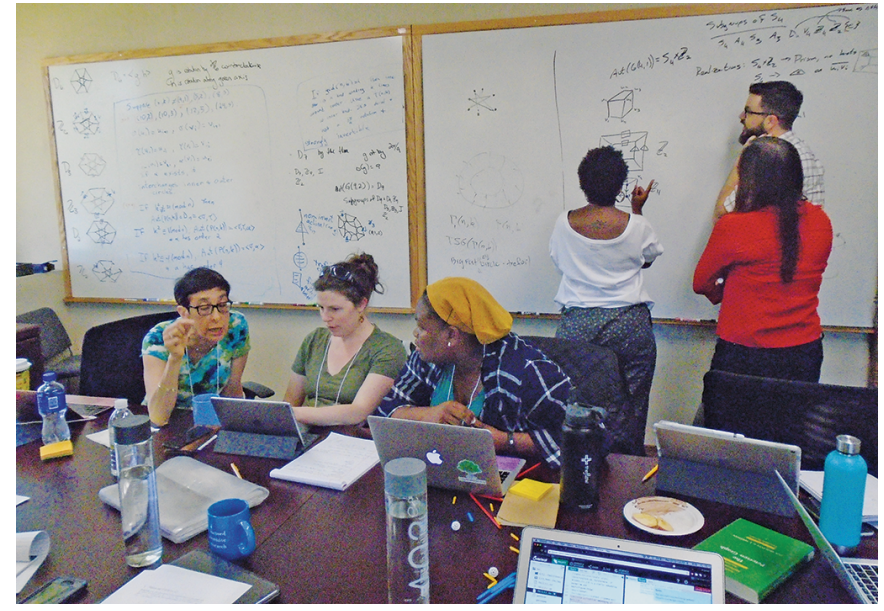

REUF participants spend most of their time doing research in groups with guidance from the project leader, as this group did at the American Institute of Mathematics in 2018.

a variety of career stages, and some use REUF to rejuvenate their research.

Some groups have continued their collaborations for many years. Members of the generalized symmetric spaces group from the 2013 REUF workshop continue their work, having met for a week in the summer multiple times, in addition to regular electronic meetings during the academic year. They have two papers published as a group and another by a group member with her undergraduate student [11]; more results are in preparation. About their participation in REUF, Jennifer Schaefer (Dickinson College) said:

I was looking for a research community to develop meaningful collaborations and hoping to expand my research into an area that was more accessible to undergraduate students. Thanks to the REUF program, I found both!

and Vicky Klima (Appalachian State University) commented:

Our monthly electronic meetings have kept me motivated to stay engaged with our projects throughout the academic year. Setting small goals that take into account our relatively high teaching and service expectations allows us to work throughout the year in a supportive environment in which no one fears letting others down. Having thought regularly about smaller questions throughout the academic year, we are prepared to jump directly into the bigger problems during our summer meetings.

Some faculty use REUF as a springboard to join an existing research community: After learning about the graph parameters zero forcing and power domination in REUF 2015, Mary Flagg (University of St. Thomas, TX) participated in the AIM workshop "Zero forcing and its applications" in January 2017 [1], where she co-authored a paper that has been published and another that is under review, in addition to two papers published with her REUF group [11]. According to Flagg:

Thanks to the 2015 REUF workshop and my group's continuation meeting in 2016, I was able to go from knowing nothing about the power domination and zero forcing problems to being totally comfortable and able to contribute in such a high level research workshop. I feel like I am now part of a new research community. This was a game-changer for me.

REUF also supports the community of those who supervise undergraduate researchers through regular communication via a listserv and two annual lunch meetings of REUF alumni at the Joint Mathematics Meetings and MAA MathFest.

Who: Faculty at undergraduate colleges and universities (about twenty per workshop).

What: One-week workshop with possible continuation meeting(s).

When: Summer (date varies).

Where: AIM (San Jose, CA) or ICERM (Providence, RI). Website: reuf.aimath.org/

Apply: Online through the host institute (the next workshop is listed on REUF website). The application is usually due in late February or early March and becomes available in December or early January.

ACKNOWLEDGMENT. The authors thank PCMI Director Rafe Mazzeo for providing information about PCMI, and AWM ADVANCE PI Kristin Lauter and Project Director Magnhild Lien for providing information about RCCWs and Research Networks.

\section{References and Further Resources}

[1] American Institute of Mathematics. Zero forcing and its applications. Available at: aimath.org/pastworkshops/ zeroforcing.htm7. Accessed May 15, 2019.

[2] The American Mathematical Society. Awards, Fellowships \& Other Opportunities. Available at: www.ams.org /opportunities. Accessed May 15, 2019.

[3] American Mathematical Society Mathematics Research Communities, 2008-2018, Available at: wWw.ams.org/programs/research-communities/ ListofMRCs2008_2018.pdf. Accessed May 15, 2019.

[4] Association for Women in Mathematics Series published by Springer (AWM-Springer Series). Proceedings of RCCWs. Information available at https://awm-math.org /publications/awm-research-volumes/ and https:// www.springer.com/series/13764?detailsPage=tit1es. Accessed May 15, 2019. 


\section{COMMUNICATION}

[5] AWM ADVANCE. Call for RCCW proposals. Available at: docs.goog7e.com/document/d/ 14cG6QiyZQaZ5Tgyq7caPb_DqQPr0R7Mo1w960sWfRbA/pub. Accessed May 15, 2019.

[6] AWM ADVANCE. Research Networks, available at: awmadvance.org/research-networks, and Research Collaboration Conferences (RCCWs), available at: awmadvance org/rccws. Accessed May 15, 2019.

[7] De Veaux R, Agarwal M, Averett M, Baumer BS, et al. Curriculum Guidelines for Undergraduate Programs in Data Science, Annual Review of Statistics and Its Applications, 4:15-30, 2017.

[8] National Science Foundation Mathematical Sciences Institutes. Available at: https://mathinstitutes.org/ institutes. Accessed May 15, 2019.

[9] Notices of the American Mathematical Society, Mathematics Opportunities section.

[10] Ott KA. Fostering Inclusive Communities: Reasons Why YOU Should Organize a Mathematics Research Community. Notices Amer. Math. Soc., 65 (2018), 1444-1445.

[11] Research Experiences for Undergraduate Faculty. Research Publications. Available at: reuf.aimath.org /outcomes. Accessed May 15, 2019.

[12] Women in Number Theory. Available at: womeninnumbertheory.org. Accessed May 15, 2019.

[13] Women in NumberTheory. Conference proceedings from WIN conferences. Available at: womeninnumbertheory .org /proceedings (only the first four are listed). Accessed June 25, 2019.

[14] Notices of the American Mathematical Society, Community Updates section.

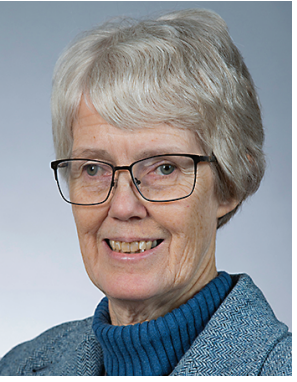

Leslie Hogben

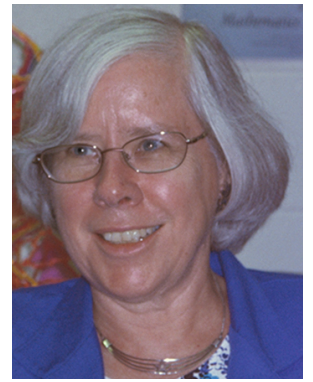

T. Christine Stevens

\section{Credits}

MRC photo is courtesy of the AMS.

PCMI photo is by Dave Titensor.

RCCW photo is by Lori Ziegelmeier.

REUF photo is by Leslie Hogben.

Photo of Leslie Hogben is by Chris Gannon, courtesy of Iowa State University.

Photo of T. Christine Stevens is by James Scott.

\section{GET MORE FROM YOUR JOURNALS}

\section{Learn about AMS MathViewer:}


$\mathrm{n}$ interactive, dual-panel reading experience for journals published by the American Mathematical Society.

AMS MathViewer's responsive HTML format perfectly renders mathematical content on desktop, tablet, or phone. Click on embedded links to formulas, theorems, figures, and references to view them in the second panel, in tandem with the original text.
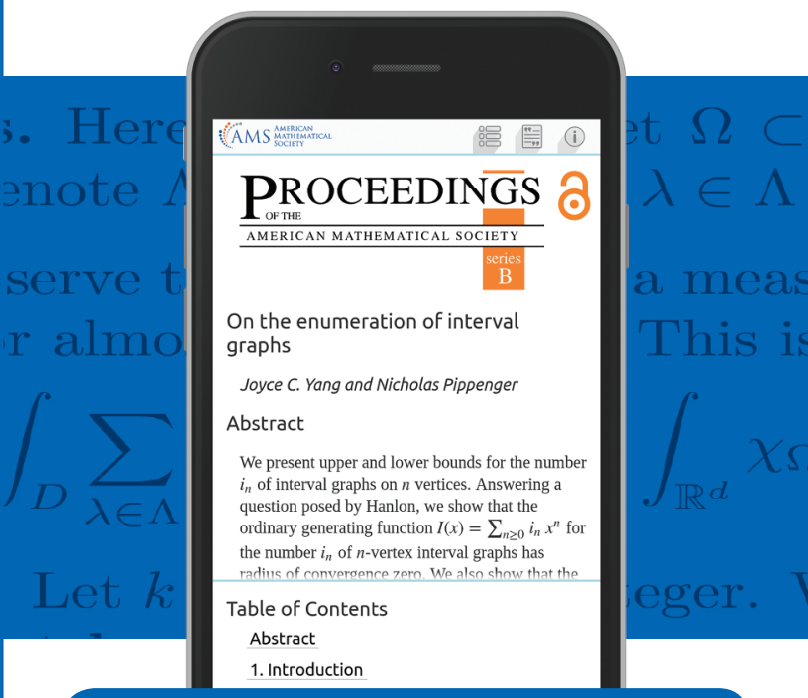

\section{CURRENTLY AVAILABLE FOR:}

Transactions of the AMS - Series B

Proceedings of the AMS - Series B

Journal of the American Mathematical Society

Mathematics of Computation

(Starting with 2019 volumes and

select articles from past issues.)

Keep an eye out for additional journals that will become available throughout the year. www.ams.org/AMSMathViewer

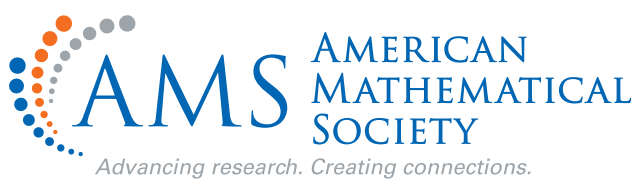

